\title{
Trade Associations: Why Not Cartels?
}

\author{
David K. Levine ${ }^{1}$, Andrea Mattozzi ${ }^{2}$, Salvatore Modica ${ }^{3}$
}

\begin{abstract}
The relevance of special interests lobbying in modern democracies can hardly be questioned. But if large trade associations can overcome the free riding problem in order to form effective lobbies, why do they not also threaten market competition by forming cartels? We argue that the key to understanding the difference lies in supply elasticity. The group discipline which works in the case of lobbying can be effective in forming a cartel only if increasing output is sufficiently costly - otherwise the incentive to deviate is too great. The theory helps organizing a number of stylized facts within a common framework.
\end{abstract}

Keywords: Cartels, labor unions, lobbying, monitoring costs, self organizing groups, special interests, trade unions.

\footnotetext{
${ }^{\star}$ First Version: February 17, 2018. We would like to thank Giacomo Calzolari, Rohan Dutta, Daniel Garcia and seminar participants at the Pamplona Jornadas Economia Industrial, the Bocconi Theory Meeting in Capri, the SAET meeting in Faro, the Fudan University Theory Workshop, the SHUFE Theory Workshop, the SJTU Theory Workshop, the LAMES meetings in Buenos Aires, and the University of the Pacific in Lima. We gratefully acknowledge support from the EUI Research Council.

Email addresses: david@dklevine.com (David K. Levine), andrea.mattozzi@eui.eu (Andrea Mattozzi), salvatore.modica@unipa.it (Salvatore Modica)

${ }^{1}$ Department of Economics and RSCAS European University Institute and Department of Economics WUSTL

${ }^{2}$ Department of Economics European University Institute

${ }^{3}$ Università di Palermo, Italy, Department of Economics, Business and Statistics (SEAS)
} 


\section{Introduction}

The objective of this paper is to contend with three stylized facts: (1) large lobbying organizations exist and are effective; (2) large cartels are uncommon and difficult to form; and (3) nevertheless trade unions that both lobby and cartelize do form and are effective. We reconcile this apparent puzzle with a simple common theory.

Our interest is in large organizations that face a free rider problem. Standard models of collusion through repetition - for example in industrial organization run afoul of the anti-folk theorem: a threat to disband a cartel when its rules are not followed cannot be effective with many firms in the presence of small noise. ${ }^{4}$ We provide an alternative model in which organizations use peer punishments to overcome free riding. In both types of models the possibility of successful collusion depends on the incentives to deviate. The greater this incentive is, the greater the punishments needed to induce compliance, and with imperfect monitoring greater punishments are more costly.

Our contention is that since the incentive to deviate in lobbying organizations is naturally limited to the gain from failing to contribute, collusion is relatively easy. In a market setting, however, large cartels are more difficult to sustain because deviation is potentially far more profitable if production can be ramped up on a large scale to take advantage of a gap between price and marginal cost. The ability to take advantage of this opportunity crucially depends on the elasticity of supply. If marginal cost rises rapidly with output then the gain from deviating is limited and collusion can be sustained. This we argue - is the case for trade unions but not the case for manufacturing firms or farms.

We emphasize that in the case of small cartels the situation is different. Small cartels are similar to lobbying organizations in that there is a natural restriction on ramping up output: individual demand is downward sloping and firms are not so eager to produce large amounts. Hence small cartels are easier to sustain.

\footnotetext{
${ }^{4}$ See Green (1982), Sabourian (1990), Levine and Pesendorfer (1995), Fudenberg, Levine and Pesendorfer (1998), Al Najjar and Smorodinsky (2001), and Pai, Roth and Ullman (2014).
} 
We do not by any means reject the traditional "small cartel" theory in which collusive arrangements are enforced by threat of future price retaliation. As we have indicated, it is for large cartels that these types of punishments are not useful. Our key point is that small cartels - those traditionally studied in the industrial organization literature - are different than large cartels. In particular we show that supply elasticity plays a much less significant role in the case of small cartels than it does for large cartels.

Going back to the stylized facts mentioned above: that large lobbying organizations exist and are effective is the core of much of the political economy of farm subsidies and trade restrictions. Certainly we observe that special business interests such as farmers, the chamber of commerce and many others are effective at lobbying government for subsidies and for entry and trade restrictions. These organizations, which we refer to as trade associations, are small as a share of the economy but often quite large in absolute size. Because of their large absolute size they face a substantial free rider problem in raising resources for lobbying: this is well documented by Olson (1965) and his successors. Still, they are able to overcome this free rider problem to be effective at lobbying. ${ }^{5}$ In the case of farming, for example, agriculture represents slightly more than $1 \%$ of U.S. GDP but there are more than 2 million farms, and they command around $0.5 \%$ of GDP in subsidies. In Japan the GDP share is similar, there are over 3 million farms and subsidies exceed $1 \%$ of GDP. ${ }^{6}$ Notice, however, that while firms benefit from lobbying, they would also benefit from collusion in the form of an output-restricting cartel. The free riding problem appears similar: produce more and reap extra profits in the cartel case, do not contribute to the lobbying effort in the lobbying case.

This raises the puzzle we address: if trade associations are so effective at

\footnotetext{
${ }^{5}$ See, for example, Grossman and Helpman (2001).

${ }^{6}$ The share of agriculture in value added is from http://data.worldbank.org/indicator/NV.AGR.TOTL.ZS. Total agricultural support as a percent of GDP is from

http: //stats . oecd. org/viewhtml . aspx?QueryId=70971\&vh=0000\&vf=0\&l\&il=\&lang=en. Data on the number of farms is from Lowder, Skoet and Raney (2016). In general, farmers are very effective at overcoming the free rider problem to lobby for farm subsidies, see for example Acemoglu and Robinson (2001).
} 
overcoming the free rider problem in order to lobby, why are they not equally effective at overcoming the free rider problem of forming a cartel? But also: how come trade unions, which also are output-restricting cartels, do typically exist? That large cartels are uncommon and difficult to form is well understood in the industrial organization literature where focus is on concentration ratios and industries with few firms - but this does not explain either the ubiquity of lobbying organizations nor the success of trade unions.

In order to understand when trade associations are successful at lobbying and at cartelization we need a theory of how they overcome free rider problems. We know from the work of Ostrom (1990) and her successors how this can be achieved: groups can self-organize to overcome the free rider problem and provide public goods through peer monitoring and social punishments such as ostracism. Formal theories of this type originate in the work of Kandori (1992) on repeated games with many players and have been specialized to the study of organizations by Levine and Modica (2016) and Dutta, Levine and Modica (2018). The basic idea is that groups choose social norms consisting of a target behavior for the group members and individual penalties for failing to meet the target; these social norms are endogenously chosen in order to advance group interests. Specifically the group designs a mechanism to promote group interests subject to incentive constraints for individual group members. It provides incentives in the form of punishments for group members who fail to adhere to the social norm. Here we build on this theory to compare the public goods problem of lobbying to that of cartelization.

Our theory helps organizing a number of stylized facts. First: we observe trade associations that lobby but do not cartelize, but rarely ones that cartelize but do not lobby. This is because the greater incentive to deviate makes cartelization less attractive than lobbying. Some trade associations both lobby and cartelize - most notably trade unions. In this case individual members are tightly constrained in how much they can increase the number of hours they work; that is, in this case marginal cost is inelastic for individual workers more than in typical production settings. The theory says that elastic marginal cost works against cartelization, while inelastic marginal cost works in favor of 
cartelization - that is, as is the case, we should not see diffuse large production cartels, but we should see trade unions cartelize.

The rest of the paper is organized as follows. In the next section we present the model. In Sections 3 and 4 we consider the cases of lobbying and large cartels, respectively. In Section 5 we argue that small cartels are different and in fact similar to lobbying organization. Section 6 deals with the efficiency of punishment and fines. We discuss a number of alternative explanations to our motivating puzzle, which appear to be not particularly compelling, in Section 7. Section 8 concludes.

\section{The Model}

We study a trade association made up of a continuum of members with unit mass. Members produce output $x$ (which given unit mass is both per capita and aggregate). The aggregate social value of the output is $V h(x)$ where we refer to $V>0$ as the value and $h(\cdot)$ is a smooth increasing function that we will define shortly.

We are interested in comparing lobbying activity and production activity. In the former case the social value is a public good. We have in mind a situation where the group engages in a lobbying effort, where $x$ is the aggregate expenditure of the group on lobbying, and where $V h(x)$ represent subsidies, or favorable laws, obtained through lobbying. The case of production applies to a market where a group of firms hold a monopoly over a good to be sold to competitive buyers. In this case output $x$ has a price, equal to marginal social value $V h^{\prime}(x)$. Letting $r(x)=h^{\prime}(x)+x h^{\prime \prime}(x)$ the marginal revenue is $\operatorname{Vr}(x)$. We assume that production cost is convex and that marginal revenue is declining with aggregate output. Specifically, on the demand side we assume the following:

Assumption. There is a satiation level $\bar{x}>0$ such that $h(x)=\bar{x}$ for all $x \geq \bar{x}$. For $x \in[0, \bar{x}]$ the function $h(x)$ is smooth with $h^{\prime}(x) \geq 0, h^{\prime \prime}(x)<0$ with the former inequality strict for $x<\bar{x}$. We also assume $r^{\prime}(x)<0$. Finally $V h^{\prime}(0)>1$.

Output - or lobbying effort - is produced at constant marginal cost normalized to 1 up to a basic capacity constraint also normalized to 1. Production 
greater than 1 is feasible but has a greater marginal cost. For example, nighttime shifts have to be added or overtime hours worked. For simplicity we assume that above basic capacity marginal cost increases linearly so that for $x>1$ marginal cost is equal to $1+\sigma(x-1)$, where $\sigma$ denotes the reciprocal of supply elasticity. The cost function for each member is therefore $C(x)=x+(\sigma / 2) \max \{0, x-1\}^{2}$.

A social norm for the group consists of a target level of output $\xi \geq 0$ and a punishment $P \geq 0$. Each group member chooses an output level $x$ and some members of the group observe a noisy binary signal of whether $x=\xi$, that is, whether the social norm was adhered to or not. ${ }^{7}$ The signal is either "good, followed the social norm" or "bad, violated the social norm." If the social norm is followed, that is, $x=\xi$, then the bad signal is generated with probability $\pi>0$. If the social norm was violated, that is $x \neq \xi$, the bad signal is generated with probability $\Pi>\pi>0$. The ratio $\pi /(\Pi-\pi) \equiv \theta>0$ is the monitoring difficulty. When the bad signal is generated the individual is sanctioned by the group and suffers a utility loss of $P$. The social norm is incentive compatible if all members find it individually optimal to follow it given that the others are doing so. The group is assumed to design an incentive compatible mechanism $\xi, P$ that maximizes the common utility of the members.

As a benchmark we define the social optimum $\chi$ as the social norm that maximizes social value minus aggregate cost, that is $V h(x)-C(x)$. This objective function is continuous and concave, with $V h^{\prime}(0)>1$ and $h^{\prime}(\bar{x})=0$. Hence the maximum is given by the unique solution to

$$
V h^{\prime}(\chi)=C^{\prime}(\chi)=1+\sigma \max \{0, \chi-1\}
$$

In the case of production this means price equal marginal cost - it is the competitive equilibrium. Cartelization consists of restricting output - in the limit to the monopoly output - so a norm close to the competitive outcome $\chi$ means the cartel effectively does not form. In the case of lobbying we take the social

\footnotetext{
${ }^{7}$ For a discussion of the peer network structure underlying this model we refer the reader to Levine and Modica (2016), Levine and Modica (2017), Levine and Mattozzi (2017) and Dutta, Levine and Modica (2018).
} 
value of output to be a public good for the group, so group effectiveness in overcoming the free-riding problem is measured by output - below $\chi$ the higher $\hat{\xi}$ the better.

For the formal analysis in the sequel we observe that the maintained continuity assumption enables us to define bounds which are used in the proofs: for $x \in[0, \bar{x}]$ we have $0<\underline{\eta} \leq\left|h^{\prime \prime}(x)\right| \leq \bar{\eta}$ and $0<\underline{\rho} \leq\left|r^{\prime}(x)\right| \leq \bar{\rho}$. These bounds depend only on $h$. We will denote by $H_{i}, i=\{1,2,3,4\}$, positive constants that depend only on $h$ (and not on $V, \sigma$ and $\theta$ ).

\section{Lobbying}

As indicated, with lobbying we take the social value of output to be a public good for the group. Hence each group member receives $V h(x)$ where $x$ is aggregate output. If a member follows the social norm by contributing $\xi$ she receives a utility $V h(\xi)-C(\xi)-\pi P$. If she deviates from the social norm the best deviation is to produce 0 and not contribute to the public good at all, resulting in utility $V h(\xi)-\Pi P$. Deviating is not optimal if and only if $C(\xi)-(\Pi-\pi) P \leq 0$. Group utility is decreasing in $P$ so for the optimal mechanism the least incentive compatible punishment $P=C(\xi) /(\Pi-\pi)$ should be used. We can then write the utility of a group member as $U(\xi)=V h(\xi)-(1+\theta) C(\xi)$; this is the group objective function.

Theorem 1. The unique optimal social norm $\hat{\xi}$ satisfies

$$
0<\chi-\hat{\xi} \leq H_{1} \min \{[\theta / V](1+\sigma), \theta+\sqrt{\theta}\}, \quad \hat{\xi}=0 \text { for } 1 \leq H_{2}[\theta / V]
$$

In summary, the result says that lobbying is absent $(\hat{\xi}=0)$ if monitoring difficulty relative to value $\theta / V$ is large; and it is successful if this is small and $\theta$ is also small (the effect of $\theta / V$ alone can be offset by large $\sigma$ ). In particular a large value $V$ is not enough to induce lobbying but other than that its effect is straightforward and unsurprising: for given $\theta$ and $\sigma$, if the prize is worthless no effort is put into obtaining it, and if it is very valuable then monitoring does 
not inhibit efficient production. ${ }^{8}$ Similar considerations apply to monitoring difficulty.

Notice that inverse supply elasticity $\sigma$ is essentially irrelevant in this context: even for given $\theta, V$ anything can happen both for small and large values of $\sigma$. In contrast we shall see that $\sigma$ is central in the cartel setting.

Proof of Theorem 1. The objective function $U$ is continuous and strictly concave on the compact set $[0, \bar{x}]$ so an optimal social norm exists and is unique and may be characterized by the derivative $U^{\prime}(\xi)=V h^{\prime}(\xi)-(1+\theta) C^{\prime}(\xi)$.

At $\xi=0$ we have $C^{\prime}(0)=1$ so the necessary and sufficient for a corner solution in which the optimal social norm is $\hat{\xi}=0$ is $V h^{\prime}(0)-(1+\theta) \leq 0$. Since $\theta / V<(\theta+1) / V$ the second condition in the theorem with $H_{2}=1 / h^{\prime}(0)$ is sufficient for a corner solution.

Suppose $V h^{\prime}(0)-(1+\theta)>0$ so the solution is interior. For $\xi \geq \chi$ we have $U^{\prime}(\xi)=V h^{\prime}(\xi)-(1+\theta) C^{\prime}(\xi) \leq V h^{\prime}(\chi)-(1+\theta) C^{\prime}(\chi)=-\theta C^{\prime}(\chi)<0$ so the optimum social norm satisfies $\xi<\chi$. Set $z=\chi-\xi>0$. We have

$$
U^{\prime}(\xi)=V h^{\prime}(\chi-z)-(1+\theta)(1+\sigma \max \{0, \chi-z-1\}) .
$$

Using $V h^{\prime}(\chi)=1+\sigma \max \{0,(\chi-1)\}$ we may write this as

$$
\begin{aligned}
U^{\prime}(\xi)= & V\left[h^{\prime}(\chi-z)-h^{\prime}(\chi)\right]+\sigma(\max \{0, \chi-1\}-\max \{0, \chi-z-1\})+ \\
& -\theta(1+\sigma \max \{0, \chi-z-1\}) \geq V \underline{\eta} z-\theta(1+\sigma \max \{0, \chi-z-1\}) .
\end{aligned}
$$

There are two cases: $z \geq \chi-1$ and $z \leq \chi-1$. In the former case we have $U^{\prime}(\xi) \geq V \eta z-\theta$ so that the necessary and sufficient condition for the social optimum $U^{\prime}(\xi)=0$ requires $V \underline{\eta} z-\theta \leq 0$ or $z \leq(1 / \underline{\eta}) \cdot(\theta / V)$. If $z \leq \chi-1$ (so that in particular $\chi>1$ ) then we have $U^{\prime}(\xi) \geq V \underline{\eta} z-\theta(1+\sigma \bar{x})$ so that $z \leq(1 / \eta)(1+\sigma \bar{x}) \theta / V$. From $V h^{\prime}(\chi)=1+\sigma(\chi-1)$ we have $V h^{\prime}(1) \geq \sigma(\chi-1)$

\footnotetext{
${ }^{8}$ This latter case, very high $V$, is less important than it might seem in the case of lobbying because special interests are small relative to the economy. So if the prize is a transfer from everyone else and it is very large, the others will have a strong incentive to lobby too and with greater resources are likely to win. As shown in Levine and Modica (2017) the rule of special interests is "do not be too greedy" because if the prize is large enough they will lose.
} 
so that $\chi-1 \leq V h^{\prime}(1) / \sigma<V h^{\prime}(0) / \sigma$. Hence

$$
\begin{aligned}
z & \leq \max \left\{\frac{1}{\eta} \frac{\theta}{V}, \min \left\{\frac{1}{\eta} \frac{\theta}{V}(1+\sigma \bar{x}), \frac{V h^{\prime}(0)}{\sigma}\right\}\right\} \\
& \leq \min \left\{\frac{1}{\eta} \frac{\theta}{V}(1+\sigma \bar{x}), \frac{1}{\eta} \frac{\theta}{V}+\frac{V h^{\prime}(0)}{\sigma}\right\} \\
& \leq \frac{1}{\eta} \frac{\theta}{V}(1+\sigma \bar{x}) \leq\left[\frac{1}{\eta} \max \{1, \bar{x}\}\right](1+\sigma) \frac{\theta}{V}
\end{aligned}
$$

giving the first half of the first condition in the theorem.

For the second half, starting from the first inequality above we can write

$$
\begin{aligned}
z & \leq\left[\frac{1}{\underline{\eta}} \bar{x}+h^{\prime}(0)\right] \min \left\{\frac{(1+\sigma) \theta}{V}, \frac{\theta}{V}+\frac{V}{\sigma}\right\} \\
& =\left[\frac{1}{\bar{\eta}} \bar{x}+h^{\prime}(0)\right]\left[\frac{\theta}{V}+\sqrt{\theta} \min \left\{\frac{\sigma \sqrt{\theta}}{V}, \frac{V}{\sigma \sqrt{\theta}}\right\}\right] \\
& \leq\left[\frac{1}{\bar{\eta}} \bar{x}+h^{\prime}(0)\right]\left(\frac{\theta}{V}+\sqrt{\theta}\right) \leq\left[\left(\frac{1}{\bar{\eta}}+h^{\prime}(0)\right) \max \left\{1, h^{\prime}(0)\right\}\right](\theta+\sqrt{\theta}),
\end{aligned}
$$

which ends the proof letting

$$
H_{1}=\min \left\{(1 / \underline{\eta}) \max \{1, \bar{x}\},\left((1 / \underline{\eta}) \bar{x}+h^{\prime}(0)\right) \max \left\{1, h^{\prime}(0)\right\}\right\} .
$$

\section{Cartels}

We now study the trade association holding a monopoly over a good to be sold to competitive buyers. Price is given by marginal social value $p(x)=V h^{\prime}(x)$ and the social optimum, characterized by $p(\chi)=C^{\prime}(\chi)$, is the competitive equilibrium. The figure below depicts the two cases where $\chi \lessgtr 1$ and shows the monopoly level of output denoted by $\mu .^{9}$

\footnotetext{
${ }^{9}$ Since we have assumed decreasing marginal revenue and weakly increasing marginal cost, $\mu$ is unique. Moreover $\mu<\chi$, since concavity of $h$ implies that marginal revenue is lower than price and hence, at $\chi$, it is also lower than marginal cost.
} 

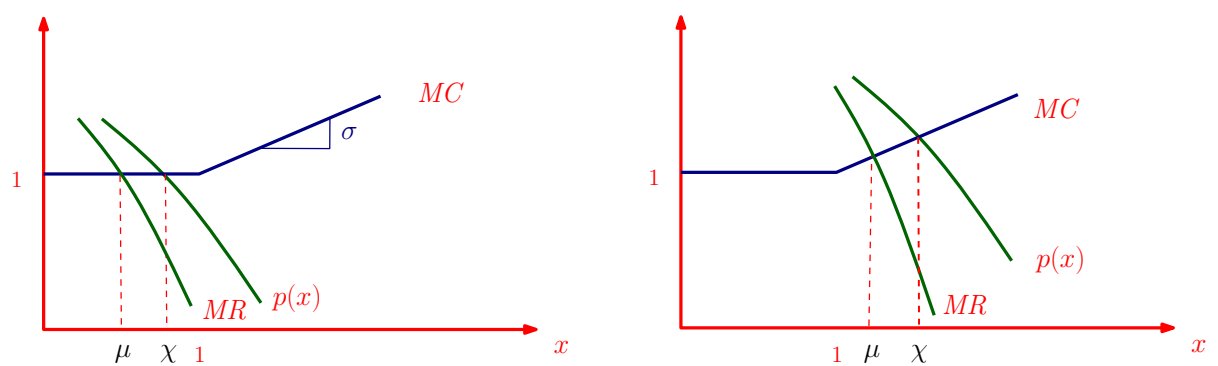

If the cartel does not form equilibrium is competitive. On the other hand no $\xi>\chi$ would be enforced since group members would be worse off than at $\chi$. Therefore we restrict attention to norms $\xi \leq \chi$. Observe that in this range $p(\xi) \geq 1$.

If industry output is $\xi$ the profits of a group member who adheres to the social norm is $p(\xi) \xi-C(\xi)-\pi P$. Denoting gross profit by $W(\xi)=p(\xi) \xi-C(\xi)$ net profits can be written as $W(\xi)-\pi P$. What is the best thing to do if violating the social norm? The key point is that the answer is not to produce $\chi$ : it is to produce more than that. Indeed, since there are a continuum of members each member is a price taker. Hence given the price $p(\xi) \geq 1$ the profit maximizing output is the highest for which marginal cost does not exceed that price. Denoting this by $\hat{x}=\hat{x}(\xi)$ we then have $\hat{x} \geq \max \{1, \chi\}$ characterized by the equality $p(\xi)=1+\sigma(\hat{x}-1)$; thus $\hat{x}(\xi)=1+(p(\xi)-1) / \sigma$. The profit from this plan is $p(\xi) \hat{x}-C(\hat{x})-\Pi P$, where $p(\xi) \hat{x}-C(\hat{x}) \geq W(\xi)$ with equality only for $\xi=\chi .^{10}$

Equating payoffs from adhering and violating the norm gives the least incentive compatible punishment $P=P(\xi)$, given by $(\Pi-\pi) P=p(\xi) \hat{x}-C(\hat{x})-W(\xi)$ that is $\pi P(\xi)=\theta[p(\xi) \hat{x}-C(\hat{x})-W(\xi)]$. Notice that $P(\xi) \geq 0$ with equality only for $\xi=\chi$; also, for $\xi<\chi$ the incentive compatible $P$ is higher the lower $\xi$ is. The expected utility from the social norm is then

$$
U(\xi)=W(\xi)-\pi P(\xi)=W(\xi)-\theta(p(\xi) \hat{x}-C(\hat{x})-W(\xi))
$$

\footnotetext{
${ }^{10}$ Proof of this: If $\xi<\chi$ then $p(\xi)>C^{\prime}(\chi) \geq C^{\prime}(\xi)$ so $p(\xi) \hat{x}-C(\hat{x})>p(\xi) \xi-C(\xi)=W(\xi)$. If $\xi=\chi$ deviation is to $\hat{x}=\max \{\chi, 1\}$; so if $\chi \geq 1$ the equality is immediate; if $\chi<1$ then $p(\chi)=1=C^{\prime}(x)$ for all $\chi \leq x \leq 1=\hat{x}$ so $p(\chi) \hat{x}-C(\hat{x})=p(\chi) \chi-C(\chi)$.
} 
Recall that the monopoly output is denoted by $\mu$ (the maximizer of $W$ ). Closeness of the norm $\hat{\xi}$ to the monopoly outcome measures success of the cartel.

Theorem 2. [Main Theorem] The optimal social norm $\hat{\xi}$ satisfies

1. $\mu<\hat{\xi} \leq \chi$, with second inequality strict if $\chi>\theta /(1+\theta)$

2. $\hat{\xi}-\mu \leq H_{3} \theta(1+[V / \sigma])$ and $\chi-\hat{\xi} \leq H_{4} / \theta(1+[V / \sigma])$

Payoff differences are similarly bounded:

3. Assuming $\chi \geq 1,[W(\mu)-U(\hat{\xi})] / W(\mu)$ has the same bounds as $\hat{\xi}-\mu$, and $[U(\hat{\xi})-W(\chi)] / W(\mu)$ has the bounds of $\chi-\hat{\xi}$.

We prove this at the end of the subsection. Note that here, provided $\chi \geq$ 1 , we also show that utility differentials have the same bounds as quantities differentials.

Monitoring difficulty goes as in the lobbying case - small $\theta$ favors cartel formation, large $\theta$ tends to prevent it. The crucial difference between the cartel and public good case is the central role $\sigma$ plays here. If $\sigma$ is small, that is supply elasticity is high, the marginal cost of exceeding basic capacity rises slowly. Hence the optimal social norm is close to competition - the cartel is not enforceable. The reason is that the temptation to cheat on the cartel is too great: in the face of a price above marginal cost it is cheap to increase output and reap a large profit. The cost of providing incentives not to take advantage of this is high: large and costly punishments must be used. The trade association does not find it in its best interest to do this.

Incidentally, the result that with low $\sigma$ the optimal norm is close to competition holds substance if it is also the case that competition is not close to monopoly. In the appendix we show that this is indeed the case.

The other interesting point is that the value $V$ works in the opposite way than in the lobbying case. A large $V$ pushes the optimal social norm towards $\chi$ in both cases. In the public good case, this is desirable for the trade association. In the case of a cartel, however, it means failure. Why does not large $V$ lead to more collusion? The answer is that on the one hand a reduction in output raises price and hence industry profits by order $V$. On the other hand it raises the per unit incentive to deviate by the same amount but also raises the amount 
a firm wants to deviate by order $V$, meaning that the incentive to deviate goes up by roughly $V^{2}$. Hence as $V$ increases the incentive to deviate goes up more than profits and so the trade association optimally restricts output less.

In short, if output elasticity is high (small $\sigma$ ) it is difficult for a trade association to self-organize, that is, to set up a cartel. For this to be possible one has to have low $\theta$ (as in lobbying), moderate $V$ (unlike in lobbying), and high $\sigma$ (no analogue in lobbying).

To be pedantic on the meaning of "no cartel forms" in the above: if $V h^{\prime}(1)>$ 1 - implying that at the competitive equilibrium marginal cost and price are greater than 1 and members of the trade association earn a competitive rent - it is always the case that $\hat{\xi}<\chi$. Hence strictly speaking a cartel always forms. The point is that if the difference is small and so is the profit gain from the cartel. Given that in practice there is certainly some fixed cost involved in organizing a cartel, it is unlikely that a cartel leading to a tiny decrease in output and yielding practically no gain in profit would be worth forming.

Proof of Theorem 2. We need to compute the derivative of $U(\xi)=(1+\theta) W(\xi)-$ $\theta[p(\xi) \hat{x}(\xi)-C(\hat{x}(\xi))]$. Since $\hat{x}$ is characterized by $p(\xi)=C^{\prime}(\hat{x})$ the derivative of the second term is just $\theta p^{\prime}(\xi) \hat{x}$. After substituting the expressions of $C^{\prime}(\xi)$ and $\hat{x}$ we then get

$$
U^{\prime}(\xi)=(1+\theta)\left[p(\xi)+p^{\prime}(\xi) \xi-C^{\prime}(\xi)\right]-\theta p^{\prime}(\xi) \hat{x}
$$

First we show that the optimal social norm satisfies $\mu<\xi \leq \chi$ with second inequality strict when $(1+\theta) \chi>\theta$. For $\xi \leq \mu$ we have $U^{\prime}(\xi) \geq-\theta p^{\prime}(\xi) \hat{x}$ so the optimum satisfies $\xi>\mu$. For $\xi>\chi$ lowering $\xi$ increases profits and relaxes the incentive constraint, so certainly $\xi \leq \chi$. Moreover when $\xi=\chi$ then $p=C^{\prime}$ and $\hat{x}=\max \{1, \chi\}$ so $U^{\prime}(\chi)=[(1+\theta) \chi-\theta \max \{1, \chi\}] p^{\prime}(\chi)$, which is strictly negative for $(1+\theta) \chi>\theta$. This proves the point 1 .

To get bounds on the social norms we start from $U^{\prime}$. Recall that $p(\xi)=$ $V h^{\prime}(\xi), p(\xi)+p^{\prime}(\xi) \xi=\operatorname{Vr}(\xi), C^{\prime}(\xi)=1+\sigma \max \{0, \xi-1\}$ and $\hat{x}=1+(p(\xi)-$ 
$1) / \sigma$. Therefore we may write

$U^{\prime}(\xi)=(1+\theta)[\operatorname{Vr}(\xi)-(1+\sigma \max \{0, \xi-1\})]-\theta V h "(\xi)\left[1+\left(V h^{\prime}(\xi)-1\right) / \sigma\right]$.

We now take $z=\xi-\mu$ and look for an upper bound on $U^{\prime}$. We have

$$
\begin{aligned}
& U^{\prime}(\xi)=(1+\theta)[\operatorname{Vr}(\mu+z)-(1+\sigma \max \{0, \mu+z-1\})]+ \\
& -\theta V h^{\prime \prime}(\xi)\left(1+\left(V h^{\prime}(\xi)-1\right) / \sigma\right) \\
& \leq-(1+\theta) V \underline{\rho} z+\theta V \bar{\eta}\left(1+\left(V h^{\prime}(0)-1\right) / \sigma\right) \text {. }
\end{aligned}
$$

This is negative for

$$
z>\frac{\theta}{1+\theta} \frac{\bar{\eta}\left(1+\left(V h^{\prime}(0)-1\right) / \sigma\right)}{\underline{\rho}}
$$

so

$$
\begin{aligned}
\xi-\mu & \leq \frac{\theta}{1+\theta} \frac{\bar{\eta}\left(1+\left(V h^{\prime}(0)-1\right) / \sigma\right)}{\underline{\rho}} \\
& \leq\left[\frac{\max \left\{h^{\prime}(0), 1\right\} \bar{\eta}}{\underline{\rho}}\right] \theta(1+[V / \sigma])=H_{3} \theta(1+[V / \sigma]) .
\end{aligned}
$$

Next take $z=\chi-\xi$ and look for a lower bound on $U^{\prime}(\xi)$. From $V h^{\prime}(\chi)=$ $1+\sigma \max \{0, \chi-1\}$ we have

$$
\begin{aligned}
U^{\prime}(\xi) & \geq(1+\theta)\left[V \underline{\eta} z+V h^{\prime \prime}(\xi)(\chi-z)\right]-\theta V h^{\prime \prime}(\xi)(\chi+V \underline{\eta} z / \sigma) \\
& =(1+\theta)\left[V \underline{\eta} z-V h^{\prime \prime}(\xi) z\right]+(1+\theta) V h^{\prime \prime}(\xi) \chi-\theta V h^{\prime \prime}(\xi)(\chi+V \underline{\eta} z / \sigma) \\
& \geq 2(1+\theta) V \underline{\eta} z-V \overline{\eta x}+\theta V^{2} \underline{\eta}^{2} z / \sigma .
\end{aligned}
$$

This is positive for

$$
z>\frac{\bar{x}}{2(1+\theta)+\theta V \underline{\eta} / \sigma}
$$

so

$$
\chi-\xi \leq \frac{\bar{x}}{2(1+\theta)+\theta V \underline{\eta} / \sigma} \leq\left[\frac{\bar{x}}{\min \{2, \underline{\eta}\}}\right] \frac{1}{\theta(1+V / \sigma)}=\frac{H_{4}}{\theta(1+V / \sigma)} .
$$


In order to bound payoff differences, first we compute a lower bound on monopoly profits: $W(\mu) \geq\left(V h^{\prime}(1 / 2)-1\right)(1 / 2)$, and the assumption $\chi \geq 1$ is equivalent to $V h^{\prime}(1) \geq 1$, so that $W(\mu) \geq\left(V h^{\prime}(1 / 2)-V h^{\prime}(1)\right)(1 / 2)=$ $\left(h^{\prime}(1 / 2)-h^{\prime}(1)\right) V / 2 \equiv \underline{W}$.

We then notice that $W(\mu)-U(\xi)>W(\xi)-U(\xi)=\pi P(\xi) \geq 0$. For the upper utility bound on $W(\mu)-U(\xi)$ observe that the optimal social norm satisfies $U(\xi) \geq U(\mu)$ so that $W(\mu)-U(\xi) \leq W(\mu)-U(\mu)$. We have $U(\mu)=W(\mu)-$ $\theta(p(\mu) \hat{x}-C(\hat{x}))$ and $p(\mu) \hat{x}-C(\hat{x}) \leq V h^{\prime}(\mu) \hat{x}-\hat{x}$. Combining these with $\hat{x}=1+$ $\left(V h^{\prime}(\mu)-1\right) / \sigma$ gives $W(\mu)-U(\xi) \leq \theta\left(V h^{\prime}(\mu)-1\right)\left(1+\left(V h^{\prime}(\mu)-1\right) / \sigma\right)$. Observe that $V h^{\prime}(\mu)-1 \leq V h^{\prime}(0)$ giving $W(\mu)-U(\xi) \leq \theta V h^{\prime}(0)\left(1+V h^{\prime}(0) / \sigma\right) \leq$ $\theta V h^{\prime}(0) \max \left\{1, h^{\prime}(0)\right\}(1+[V / \sigma])$. Dividing by the lower bound on monopoly profits $\underline{W}$ gives the bound in the theorem:

$$
\frac{W(\mu)-U(\xi)}{W(\mu)} \leq\left[\frac{2 h^{\prime}(0) \max \left\{1, h^{\prime}(0)\right\}}{h^{\prime}(1 / 2)-h^{\prime}(1)}\right] \theta(1+[V / \sigma]) .
$$

Next, $U(\xi)-W(\chi)>0$ since $P(\chi)=0$. For the upper utility bound observe that the utility gain from $\xi$ over $\chi$ is less than the profit gain because $P(\xi)$ gets larger as $\xi$ goes down. Reducing output by $\chi-\xi$ raises price by no more than $\bar{\eta} V(\chi-\xi)$ and saves at most marginal cost times $\chi-\xi$ and that marginal cost is at most $V h^{\prime}(1)$. Hence $U(\xi)-W(\chi) \leq V\left(\overline{\eta x}+h^{\prime}(1)\right)(\chi-\xi)$, and dividing by $\underline{W}$ we get

$$
\left.\frac{U(\xi)-W(\chi)}{W(\mu)} \leq 2 \frac{\overline{\eta x}+h^{\prime}(1)}{h^{\prime}(1 / 2)-h^{\prime}(1)}\right)(\chi-\xi)
$$

Remark. We have not dealt here with the possibility of entry. Notice that the considerations with entry are similar to those discussed here: in the case of lobbying a modest level of entrants who do not belong to the organization will have a modest effect on its effectiveness. In the case of cartelization the effect of a modest level of entrants who do not belong to the organization will depend on how capacity constrained the entrants are: that is how large is $\sigma$. If $\sigma$ is large then the entrants will not have a big impact on the price set by the cartel, while if $\sigma$ is large they will. 


\section{Small Cartels Are Different}

The theory we have presented is a theory of large cartels: cartels where individual firms are sufficiently small that their individual output has no impact on prices. Existing theory - and practice - has focused on small cartels. Here we present a brief overview of that theory, not to prove new results but to give a clear idea how the theory differs from the theory of large cartels. There are two dimensions in which the theory is different. The first is that information can be used to enforce collective punishments when cartels are small. As we will indicate this can be enormously advantageous for the cartel. The second dimension is that firms face downward sloping individual demand curves: while with large cartels the only restraint on deviation lies in increasing cost, with small cartels firms limit their deviation because their increased output lowers price. For this reason, cost conditions - $\sigma$ - plays a much less important role.

For reasons of comparability will will retain the same market structure as in the large cartel case. We will continue to assume the cartel sets a quota $\xi$, that if $\phi$ represents per firm output industry price is given by $p(\phi)=V h^{\prime}(\phi)$ and that the cost to a firm of producing $x$ is given by $C(x)=x+(\sigma / 2) \max \{0, x-1\}^{2}$. Contrary to what we assumed earlier, however, now there is a fixed finite number of firms $N$. The key feature is that if $x$ is the output of an individual firm and the other firms adhere to the quota $\xi$ then per firm output is $\phi=[(N-1) \xi+x] / N$.

\section{Information and Collective Punishment}

We first make a simple observation: if per firm output is observable then it is possible to use a collective punishment $P_{c}$ to punish all firms equally if $\phi \neq \xi$. In a dynamic setting this might take the form of a price war as often studied in the literature. With sufficiently large $P_{c}$, corresponding to sufficient patience on the part of the firms in the dynamic case, all deviations can be made unprofitable. Hence neither firm will wish to deviate, punishment never occurs on the equilibrium path so has no social cost, and any social norm is incentive compatible. In particular the cartel will form and agree on the monopoly solution $\hat{\xi}=\mu$ and the firms will split the monopoly profit. If the actual output of a firm $\tilde{x}=x+\epsilon$ is a noisy function of intended firm output $x$ it can be shown 
that if the noise is sufficiently small (see, for example Green and Porter (1984)) the cartel will form and nearly the monopoly profit will be split between the firms.

However: with noise this method does not scale to large cartels. As the number of firms increases individual deviations being small can no longer easily be distinguished from the background noise and as a result individual firms have little effect on the probability of punishment and so have every incentive to deviate. Even if the individual firm outputs $\tilde{x}$ are observed this is no help: see Levine and Pesendorfer (1995) and Fudenberg, Levine and Pesendorfer (1998) for a proof of this. It is for this reason that our analysis of large cartels focuses on the combination of individual signals and individual punishments. As this case is of interest also in small cartels, we turn to this next.

\section{Demand and Cost}

We now drop the assumption that output is directly observable, and return to the information structure of the original model: if $x=\xi$ then a bad signal is generated with probability $\pi>0$, while if $x \neq \xi$ a bad signal is generated with probability $\Pi>\pi>0$. We consider two cases: an individual punishment $P$ and a collective punishment $P_{c}$. In the former case we define as before $\theta=\pi /(\Pi-\pi)$. In the latter case there are different schemes $m$ that may be used for determining how the triggering of the collective punishment depends on the number of bad signals. Let $\pi(m)$ be the probability that punishment occurs when no firm deviates and $\Pi(m)$ the probability of punishment when 1 firm deviates. We define $\theta(m)=N \pi(m) /(\Pi(m)-\pi(m))$ and $\theta=\min _{m} \theta(m)$. A key point is that from the bounds given in Levine and Pesendorfer (1995) with collective punishment $\theta \rightarrow \infty$ as $N \rightarrow \infty$. On the other hand, with individual punishment $\theta$ is independent of $N$ which is why individual punishment is essential for large cartels.

The next result shows that in this context we can bound the distance from cartel output to monopoly independently of $\sigma$ :

Theorem 3. $\hat{\xi}-\mu \leq H_{5} \theta(N-1)$.

This bound is obviously useful only for small cartels - that is for $N$ small. 
The idea here is a simple one: regardless of $\sigma$ - even if it is zero - downward sloping demand implies a limit to how much output firms want to produce when they deviate and hence a limit to how much they can gain by deviating. This strongly contrasts with the large cartel case where large enough $\sigma$ drives the cartel solution towards competition.

In fact the bound is valid for collective punishment as well as individual punishment - although indeed, $\theta$ is larger with collective punishment and grows with $N$. Never-the-less in the small cartel case if it is necessary to use collective punishment such as a price war, there is no bar to doing so, and many papers in the small cartel literature focus on that case.

Proof. First we observe that $\hat{x}$ is bounded independent of $\sigma$ : indeed we have $\hat{x} \leq N \bar{x}$ as the individual firm will never choose industry output to be above the satiation level. Next we look at the objective function

$$
U(\xi)=(1+\theta) W(\xi)-\theta[p([(N-1) \xi+\hat{x}(\xi)] / N) \hat{x}(\xi)-C(\hat{x}(\xi))]
$$

By the envelope theorem the derivative is

$U^{\prime}(\xi)=(1+\theta)\left[p(\xi)+p^{\prime}(\xi) \xi-C^{\prime}(\xi)\right]-\theta p^{\prime}(((N-1) \xi+\hat{x}(\xi)) / N) \hat{x}(\xi)(N-1) / N$

In the proof of Theorem 2 we showed this results in a bound of the form

$$
\hat{\xi}-\mu \leq\left[\frac{\max \left\{h^{\prime}(0), 1\right\} \bar{\eta}}{\underline{\rho}}\right] \theta \hat{x}(N-1) / N \leq\left[\frac{\max \left\{h^{\prime}(0), 1\right\} \overline{\eta x}}{\underline{\rho}}\right] \theta(N-1)
$$

giving the desired result.

\section{Efficiency of Punishment and Fines}

We have so far assumed that the punishment $P$ is a cost to the cartel of punishing a bad signal. This would be the case if the punishment involves some form of exclusion. In practice, however, cartels often use fines rather than exclusion: a fine is not a net cost to the cartel, since the cost to the member punished is a gain to the other members. In this case, the cost of punishment 
to the cartel is $\psi P$ where $\psi$ is a measure of the inefficiency of the punishment. In the extreme case of a pure transfer payment $\psi=0$; in the case that the cost to the member punished is the cost to the cartel - the case we considered earlier - $\psi=1$. If there are additional costs to other cartel members of exclusion we might also have $\psi>1$.

If we consider this more general model, the first observation is that the basic theory changes quantitatively but not qualitatively. That is, we have defined monitoring difficulty as $\theta \equiv \pi /(\Pi-\pi)$. We can incorporate the inefficiency of punishment by defining instead $\theta \equiv \psi \pi /(\Pi-\pi)$ and the earlier results remain valid. We see immediately that a more efficient punishment technology - such as fines - improve possibilities for cartelization. This idea that transfer payments enhance collusion appears in the repeated game literature - it is the basis for the folk theorem with imperfect private information where patience allows future punishments to take the form of efficient transfer payments rather than inefficient pure punishments. This is discussed in Fudenberg, Levine and Maskin (1994). In the mechanism design literature, see for example D'Aspremont and Gerard-Varet (1979), the same idea is captured through budget balance. However, as Myerson and Satterthwaite (1983) showed not all information technologies allow the first best to be obtained with budget balance so it can be optimal to "burn money", corresponding to socially costly punishments. ${ }^{11}$

Fines are not a panacea. First, fines must ultimately be backed by some other more costly form of punishment - the punishment for refusing a fine cannot simply be a larger fine that will also be refused. Second, transfer payments while more efficient than pure punishment generally are not 1-1 - that is there are generally costs and inefficiencies of collecting fines - as with taxes - and the value to the recipient will generally be less than the cost to the payer. Finally, fines introduce malincentive for monitoring. Those who receive the fines have an

\footnotetext{
${ }^{11}$ Notice that fines are an individual not a collective punishment. As we have observed this is useful in a cartel of any size. This point does not always come across clearly in empirical studies: a good example is Hyytinen, Steen and Toivanen (2018) who study legal cartels in Finland. One piece of evidence they use to show that legally registered cartels are not merely paper entities is the use of fines. On the other hand the theory on which their study is based is that of repeated games and price wars as punishment.
} 
incentive to make false accusations. ${ }^{12}$ Indeed, if any cartel member can secretly plant false evidence - switch a signal to a bad signal - then with fines all will choose to do so and the signal will be useless. All of these considerations lead to the conclusion that while we may get a substantial reduction in $\psi$ by using fines, it is unlikely to be zero.

There are two key conclusions: first, if fines are available they are likely to be used, and second, if fines are available cartelization is more likely. In particular in the case of large cartels, high supply elasticity $\sigma$ may be offset by a low value of $\psi$. A case in point is that of the Consorzio Grana Padano, that owns the trademark for Grana Padano cheese in Italy. It is a reasonably large group consisting of about 200 producers and collects fees from farmers and monitors quality of cheese production before authorizing the use of the trademark. ${ }^{13}$ The Consorzio was fined by the Italian Competition Authority (decision 4352) essentially for imposing fines to members producing too much. ${ }^{14}$ The Consorzio has very good monitoring technology (there are inspectors on the floor of the producers) and they believed that their system of fines was legal. Hence in this case $\theta$ was quite small both because $\pi$ is small and because $\psi$ is small. The cartel activities were also very visible and as a result anti-trust action was effective. The general implication for anti-trust authorities is that since fines are easy to observe, illegalizing them should be an effective means of reducing cartelization.

In conclusion,cost elasticity is not so likely to inhibit the formation of large cartels when fines can be used as punishment. When membership in the association is crucial it will be relatively easy to enforce fines. Two contexts in which this is true are trademark associations and sports leagues. We do not think it is a coincidence that sports leagues which can very easily fine their members lobby

\footnotetext{
${ }^{12}$ We see this, for example, when local governments lower speed limits and reduce the length of yellow lights in order to increase revenue from traffic fines. Indeed the problem of false accusations is an ancient one - one element of the code of Hammurabi is punishment for false accusation - see Fudenberg and Levine (2006).

${ }^{13}$ See https://www.granapadano.it/public/file/201904USA-34282.pdf

${ }^{14}$ See Consorzio Grana Padano, 24 June 2004, n. I569, Bulletin 26/2004, and Siragusa, Beretta and Bay (2019).
} 
heavily and in many cases successfully for exemption from anti-trust laws.

\section{Discussion}

In this section we discuss a number of alternative explanations to our motivating puzzle. First, it could be argued that monitoring is more difficult in a cartel setting than in a public goods setting, hence a collusive arrangement more difficult to enforce. However, it is not immediately obvious that farmers living in a farm community are less able to observe how many fields their neighbors plant than they are to observe whether their neighbors contribute to farm lobbying efforts. In manufacturing monitoring of prices is difficult, and perhaps even monitoring of outputs. But monitoring of inputs is not so difficult. If manufacturing firms agreed to limit themselves to one six hour shift a day - in respect of workers rights - that would not only be relatively easy to monitor but would be unlikely to violate anti-trust laws.

Second, it could be argued that public policy and anti-trust law play a role in inhibiting cartel formation but are not directed against lobbying. However, most anti-trust activity is directed against small cartels: for example the average number of firms in a cartel pursued by the European Commission is 7.61 (see Ordóñez-De-Haro, Borrell and Jiménez (2018)). As we have observed input restrictions are not so likely to run afoul of anti-trust laws - manufacturing firms can hide collusion as concern over workers rights. In a similar way if farmers got together and talked about colluding to reduce output this would be legally problematic. But if they get together - as they do - to discuss best farming practices and agree that a number of fields should be left fallow, that less fertilizers and less intensive farming is a better practice - and this could be successfully enforced as it is in the case of contributions to lobbying efforts - it seems unlikely it would run afoul of anti-trust policy. Indeed, most governments encourage farmers to discuss and adapt best farming practices - often even subsidize them to do so. Finally, even when cartels were legal, the existing empirical evidence seems to support the idea that large cartels were not very common. For example Haucap, Heimeshoff and Schultz (2010) documents that the median number of members for legal cartels authorized by the German 
Federal Cartel Office (FCO) between 1958 and 2004 was four. ${ }^{15}$ Our view is that while legal restrictions may be part of the answer it is unlikely to be the entire answer.

Third, it must be observed that some industries with a large number of "firms" do indeed successfully restrict output. Workers are often able to exploit their monopsony power, especially in a labor union setting. Even without a labor union an informal agreement not to "work too hard" is common. Since the demand for effort is downward sloping, workers as a group can take advantage of their monopsony power by reducing effort - and indeed they often do exactly that. Furthermore, while nowadays we think of labor unions as encouraged by and supported by government this has not always been true. In the early 20th Century labor unions were actively discouraged by governments - and indeed union members were sometimes murdered as happened, for example, in 1927 at the Columbine mine massacre (Zieger (1994)). More recently the Solidarity Union in Poland operated in a hostile political environment. Nevertheless unions were effective in restricting input.

Finally, it may be that successful lobbying alleviates the need for cartels. This could be the case of price support or output limitation schemes that we sometimes see in agriculture. It is not true for direct subsidies which are also common in agriculture. However, the most common form of successful lobbying are restrictions on competition - illegalizing domestic competitors, for example in the case of taxis, or more commonly limitations on foreign competition. These types of lobbying success raise rather than lower then incentive for cartelization by increasing monopoly power. Moreover, it should not escape attention that lobbying is endogenous: those organizations that we find would most easily be able to cartelize - labor unions, sports leagues - should lobby for legal cartelization. Organizations such as manufacturing firms that would find it difficult to cartelize should lobby instead for restrictions on competition. This seems to be the case.

\footnotetext{
${ }^{15}$ Interestingly, the median number of members of illegal cartels in the same period was five.
} 


\section{Conclusion}

In practice trade associations can both lobby and form cartels and must allocate resources between the two. Our goal here is to understand a simpler and more conceptual point: what is the difference between the free rider problem in lobbying and in forming a cartel? We have used the same model for both lobbying and cartel formation in order to isolate the effect of market organization. There is no reason to presume that the technology for producing resources to be used for lobbying is the same as market production technology. What our results show, however, is that this is probably not an important reason why lobbying is so much more common than cartel formation. In particular, the value of the prize or market plays little role in cartel formation. While it plays an important role in lobbying (with more valuable prizes likely to elicit greater lobbying effort) there is reason to think that the size of the prize is limited in practice by opposition from those who have to pay the subsidy (Levine and Modica (2017)). Rather, our results direct attention to two key variables: the difficulty of monitoring and the elasticity of supply.

The difficulty of monitoring plays a key role in both lobbying and cartel formation: if monitoring is difficult then public goods such as lobbying and cartels will not be provided by trade associations. It may be that there are important differences in these costs between lobbying efforts and cartel formation - but it is neither obvious nor evident that this is the case. The second key variable is supply elasticity. We find this unimportant in lobbying but crucial in cartel formation. If it is relatively low cost to increase output, incentives to cheat on a cartel are great and cartel formation will be inhibited. If - by contrast - it is difficult to increase output beyond basic capacity the cartel formation problem is relatively similar to the lobbying problem.

We can illustrate our results by contrasting three industries:

1. Manufacturing firms: it is relatively easy for manufacturers to observe each others activities but firms can easily expand in size by hiring more inputs.

2. Plant workers: it is relatively easy for workers on a factory floor to observe each others effort but workers are physically limited in how much they can increase individual output. 
3. Hair dressers: like plant workers hair dressers are physically limited in how much they can increase individual output, but they are diffused in many locations and cannot easily monitor each other.

The theory then predicts the pattern given in the table below, which is indeed what we observe: manufacturers should be effective at lobbying but not cartelization, plant workers at both, and hair dressers at neither.

\begin{tabular}{c|cccc} 
industry & monitoring cost & supply elasticity & lobbying & cartel \\
\hline manufacturing & low & high & yes & no \\
plant workers & low & low & yes & yes \\
hair dressers & high & low & no & no
\end{tabular}




\section{Appendix: Competition versus Monopoly}

In the text we established bounds on how close the optimal social norm is to the competitive and monopoly output. Of course the two could be close to each other. We give conditions under which the two are different in both quantity and profit:

Theorem 4. The output and profit differences between competition and monopoly have the following bound:

$$
\chi-\mu, \frac{W(\mu)-W(\chi)}{W(\mu)} \geq H_{5} \min \{\chi, 1\}\left(\frac{1}{1+[\sigma / V]}+\max \left\{0, \frac{1}{V}-r(1)\right\}\right) .
$$

The case in which $V h^{\prime}(0)$ is very close to 1 is uninteresting. In this case $\chi$ is close to zero, the market is not very profitable and little is produced regardless of how the industry is organized. Otherwise for the competitive and monopoly solutions to be close together two things must both be true: $\sigma / V$ must be very large and marginal revenue at aggregate output 1 equal to $\operatorname{Vr}(1)$ must be either greater than marginal cost or at least not too much smaller. This corresponds in the limit to the well known case of inelastic supply and marginal revenue above marginal cost at the capacity constraint. In this case there is no difference between monopoly and competition - the monopolist is content to take competitive rents rather than profits.

An especially important point is that when it is easy to increase output above basic capacity, that is, $\sigma$ is small, then not only is the optimal social norm close to the competitive equilibrium in quantity and profit - but the competitive equilibrium is not close to monopoly either in quantity or profit.

Proof. First consider $V h^{\prime}(1) \leq 1$ (case $\left.\chi \leq 1\right)$. Marginal revenue at the competitive equilibrium is $\operatorname{Vr}(\chi)=p(\chi)+p^{\prime}(\chi) \chi \leq V(1-\underline{\eta} \chi)$. Hence from $\operatorname{Vr}(\mu)=1$ we deduce that it must be $\chi-\mu \geq[\underline{\eta} / \bar{\rho}] \chi$. So the result holds for $V h^{\prime}(1) \leq 1$.

For $V h^{\prime}(1)>1$, that is $\chi>1$ we will show

$$
\chi-\mu \geq \frac{\underline{\eta} / 2}{\sigma / V+\bar{\rho}+\bar{\eta}}+\frac{\max \{0,1 / V-r(1)\}}{2 \bar{\rho}}
$$


from which the result follows.

Since $\chi-\mu=\chi-1+1-\mu$ we bound the two terms. First suppose that $r(1) \leq 1 / V$. Then at $x=1-[1-V r(1)] / V \bar{\rho}$ we have $\operatorname{Vr}(x) \leq 1$ so that $x \geq \mu$, or $1-\mu \geq[1-V r(1)] / V \bar{\rho}$. Next we bound $\chi-1$. Recall that $V h^{\prime}(\chi)-(1+\sigma(\chi-1))=0$. This implies $V h^{\prime}(1)-V \bar{\eta}(\chi-1)-(1+\sigma(\chi-1)) \leq 0$, from which

$$
\chi-1 \geq \frac{V h^{\prime}(1)-1}{\sigma+V \bar{\eta}} .
$$

For $r(1)>1 / V$ we add together the bounds on $1-\mu$ and $\chi-1$ to find

$$
\begin{gathered}
\chi-\mu \geq \frac{h^{\prime}(1)-1 / V}{\sigma / V+\bar{\eta}}+\frac{(1 / V)-r(1)}{\bar{\rho}} \geq \frac{1}{2} \frac{h^{\prime}(1)-1 / V}{\sigma / V+\bar{\eta}}+\frac{(1 / V)-r(1)}{\bar{\rho}} \\
\geq \frac{1}{2} \frac{h^{\prime}(1)-r(1)}{\sigma / V+\bar{\eta}+\bar{\rho}}+\frac{1}{2} \frac{(1 / V)-r(1)}{\bar{\rho}} .
\end{gathered}
$$

Moreover, $r(1) \leq h^{\prime}(1)-\underline{\eta}$. This covers the case $r(1)>1 / V$.

Now suppose that $r(1) \leq 1 / V$. By the intermediate value theorem for $x \leq y \leq \chi$ we have

$$
\begin{aligned}
V r(x) & =V r(\chi)-V r^{\prime}(y)(\chi-x) \leq p(\chi)-V \underline{\eta} \chi+V \bar{\rho}(\chi-x) \\
& \leq 1+\sigma(\chi-1)-V \underline{\eta}+V \bar{\rho}(\chi-x)
\end{aligned}
$$

and $C^{\prime}(x) \geq 1+\sigma(1-x)$. Hence if $1+\sigma(\chi-1)-V \underline{\eta}+V \bar{\rho}(\chi-x)-(1+\sigma(x-1))=0$ then $\mu \leq x$. Solving the equality for $x$ gives the bound

$$
\chi-\mu \geq \frac{\underline{\eta}}{\sigma / V+\bar{\rho}} \geq \frac{1}{2} \frac{\underline{\eta}}{\sigma / V+\bar{\eta}+\bar{\rho}} .
$$

For the profit bound we have

$$
W(\mu)-W(\chi)=\int_{\mu}^{\chi}\left(-V r(x)+C^{\prime}(x)\right) d x
$$


Choose $y=\mu+(\chi-\mu) / 2$ we have

$$
W(\mu)-W(\chi) \geq \int_{y}^{\chi}\left(-\operatorname{Vr}(x)+C^{\prime}(x)\right) d x .
$$

Moreover $-\operatorname{Vr}(\mu)+C^{\prime}(\mu)=0$ implies for $x \geq y$ that $-\operatorname{Vr}(x)+C^{\prime}(x) \geq$ $V \underline{\rho}(\chi-\mu) / 2$ giving the desired bound.

\section{References}

Acemoglu, D. and J. Robinson (2001): "Inefficient Redistribution," American Political Science Review 95: 649-661.

Al-Najjar, N. I. and R. Smorodinsky (2001): "Large nonanonymous repeated games," Games and Economic Behavior 37: 26-39.

Dutta, R., D. K. Levine and S. Modica (2018): "Peer Monitoring, Ostracism and the Internalization of Social Norms," EUI.

d'Aspremont, Claude, and Louis-André Gérard-Varet: "Incentives and incomplete information (1979):" Journal of Public Economics 11: 25-45.

Fudenberg, D. and D. K. Levine (2006): "Superstition and Rational Learning," American Economic Review 96 (3): 630-651.

Fudenberg, D., D. K. Levine and E. Maskin (1994): "The Folk Theorem with Imperfect Public Information," Econometrica, 62(5): 997-1039.

Fudenberg, D., D.K. Levine and W. Pesendorfer (1998): "When are nonanonymous players negligible?" Journal of Economic Theory, 79: 46-71.

Green, E. J. (1982): "Noncooperative price taking in large dynamic markets," In Noncooperative Approaches to the Theory of Perfect Competition: 37-64.

Green, E. J. and R. H. Porter (1984): "Noncooperatove Collusion Under Imperfect Price Information," Econometrica 52(1): 87-100.

Grossman, G. M., and E. Helpman (2001): Special interest politics, MIT press

Hyytinen, A., F. Steen, and O. Toivanen (2018): "Cartels Uncovered," American Economic Journal: Microeconomics 10: 190-222.

Haucap, J., U. Heimeshoff and L. M. Schultz (2010): "Legal and Illegal Cartels in Germany Between 1958 and 2004," No 08, DICE Discussion Papers, University of Düsseldorf.

Kandori, M. (1992): "Social norms and community enforcement," The Review of Economic Studies 59(1): 63-80.

Levine, D. K. and A. Mattozzi (2017): "Voter Turnout with Peer Punishment," EUI

Levine, D. K.and S. Modica (2016): "Peer Discipline and Incentives within Groups", Journal of Economic Behavior and Organization 123: 19-30 
Levine, D. K.and S. Modica (2017): "Size, Fungibility, and the Strength of Lobbying Organizations", European Journal of Political Economy 49: 71-83

Levine, D. K. and W. Pesendorfer (1995): "When are agents negligible?" American Economic Review.

Lowder, S. K. , J. Skoet, and T. Raney (2016): "The Number, Size, and Distribution of Farms, Smallholder Farms, and Family Farms Worldwide," World Development 87.

Myerson, Roger B., and Mark A. Satterthwaite (1983): "Efficient mechanisms for bilateral trading," Journal of Economic Theory 29: 265-281.

Olson Jr., M. (1965): The Logic of collective action: public goods and the theory of groups, Harvard Economic Studies.

Ordóñez-De-Haro, J., R. Borrell and J. L. Jiménez (2018): "The European Commission's Fight Against Cartels (1962 - 2014): A Retrospective and Forensic Analysis," Journal of Common Market Studies 56, 1087-1107.

Ostrom, E. (1990): Governing the commons: The evolution of institutions for collective action, Cambridge university press.

Pai, M. M., A. Roth and J. Ullman(2014): "An anti-folk theorem for large repeated games with imperfect monitoring," arXiv preprint arXiv:1402.2801.

Sabourian, H. (1990): "Anonymous repeated games with a large number of players and random outcomes," Journal of Economic Theory, 51(1), 92-110.

Siragusa, M., M. Beretta, and M. Bay (2009): Competition Law in Italy: The first 20 years of law and practice, Cleary Gottleib.

Zieger, R. H. (1994): American workers, American unions, Johns Hopkins Univ Press. 